\title{
Implementation and Operation of a Cloud-Based Participatory Damage Recognition System to Obtain a Common Operational Picture that Supports a Quick Disaster Response
}

\author{
${ }^{1}$ Reo Kimura, ${ }^{2}$ Munenari Inoguchi, ${ }^{2}$ Keiko Tamura, ${ }^{3}$ Yuji Nawa, ${ }^{4}$ Haruo Hayashi \\ ${ }^{1}$ University of Hyogo, ${ }^{2}$ Niigata University, ${ }^{3}$ ESRI, ${ }^{4}$ Kyoto University
}

\begin{abstract}
A cloud-based participatory damage recognition survey system using WEB-GIS was implemented and operated to collect comprehensive and fundamental information that could be utilized to generate a common operational picture, which are essential for the recovery and reconstruction efforts during widearea, complex disaster responses. The extent of damages due to buildings that were washed away by the tsunami that occurred during the Great East Japan (Tohoku) Earthquake of 2011 was captured using aerial views. The objective of this research was to develop the cloud-based participatory WEB-GIS system constructing the common operational picture in order to facilitate the decision-making process of disaster response. The team set the research aim to create the web service of certifying property damage due to Tsunami. Using developed service the team conducted the demonstration experiment to survey to identify buildings that were washed away in the three afflicted prefectures of Iwate, Miyagi, and Fukushima by Tohoku Earthquake.
\end{abstract}

\section{Introduction}

High-quality disaster responses cannot be provided without constructing a common operational picture (COP) and organic collaboration among stakeholders. To this end, it is effective to visualize damages and responses using a Geographic Information System (GIS), which facilitates decision-making based on visualized data. In reality, however, during the chaotic days immediately following a disaster, the collection and consolidation of data for visualization is insufficient. The absence of data to be visualized hinders our efforts to construct a common operational picture.

\section{2011 Great East Japan Earthquake}

The Great East Japan (Tohoku) Earthquake on March 11, 2011 generated massive tsunamis that washed away many buildings (Figure 1). Approximately 20,000 people died or went missing, approximately 130,000 buildings were severely damaged or washed away, and approximately 255,000 buildings sustained some

damages. The amount of direct damages was estimated to be between 16 and 25 trillion yen and the amount of debris exceeded 26.7 million tons. As many as 73,500 people were evacuated from the damaged prefectures (Table 1). Japan defined the responsibilities of the national and regional governments during disaster responses in the Fundamental Disaster Relief Act, which was implemented in 1961. The Tohoku Earthquake was a huge disaster that shook the entire nation (Figure 2). This disaster inflicted severe human and property

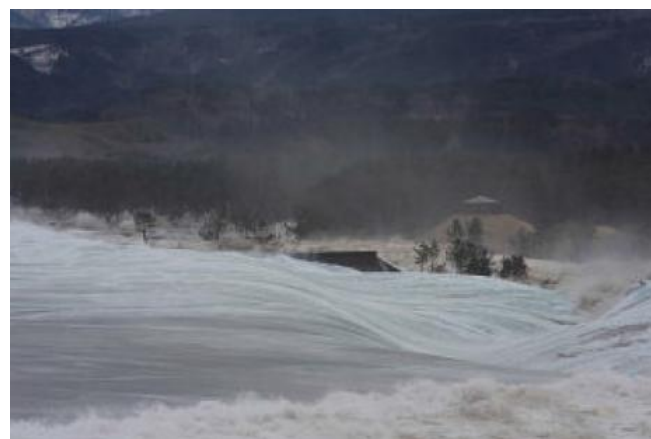

Figure 1. The 2011 Great East Japan (Tohoku) Earthquake and Tsunami $(\mathrm{Mw}=9.0)$

Noda village, Iwate prefecture, Japan

Table 1. Summary of the impact at the Great East Japan Earthquake in 2011

\begin{tabular}{|c|c|c|}
\hline & & Impact \\
\hline \multirow{2}{*}{ Mortality } & Dead & 15,854 \\
\hline & Missing & 3,155 \\
\hline \multirow{2}{*}{$\begin{array}{l}\text { Housing } \\
\text { Damage }\end{array}$} & Severe & 129,101 \\
\hline & Moderate & 254,057 \\
\hline \multicolumn{2}{|c|}{ Direct Losses ( in Trillion Yen) } & $16-25$ \\
\hline \multicolumn{2}{|c|}{ Debris (in ton) } & $26,700,000$ \\
\hline \multicolumn{2}{|c|}{$\begin{array}{l}\text { Displaced Victims } \\
\text { (left their prefectures) }\end{array}$} & 73,466 \\
\hline \multicolumn{2}{|c|}{ Disaster Type } & Landscape-scale \\
\hline
\end{tabular}

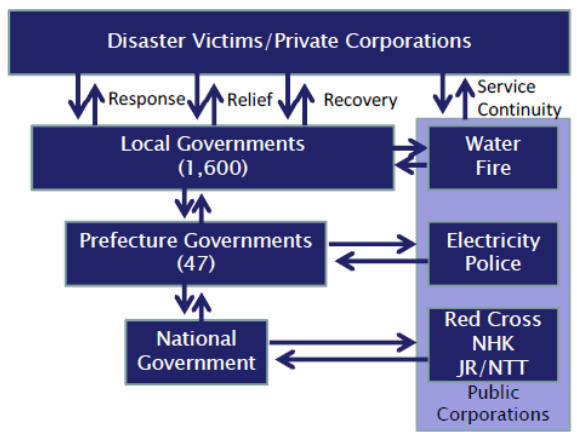

Figure 2. Japanese Disaster Management System since 1961 
damages to 3 out of 47 prefectures and 22 prefectures established disaster response centers.

\section{Efficient methods of earthquake and tsunami damage estimation}

Our research team initially organized building point data according to altitude in a geographic space to create a big picture of damages. We then estimated the number of damaged buildings in each region alongside the height of the tsunamis in these regions (as reported by the Japan Meteorological Agency). The results indicated that 270,000 buildings were damaged in three prefectures of Iwate, Miyagi, and Fukushima. Figure 3 shows the results of our information analyses, which were shared with the disaster response workers. Under ideal circumstances we would have conducted a field survey to verify the results of our estimate. However, immediately after the earthquake, access to the disaster area was limited and the survey would have taken a very long time. These conditions necessitated the development of an efficient survey method that did not require visits to the fields.

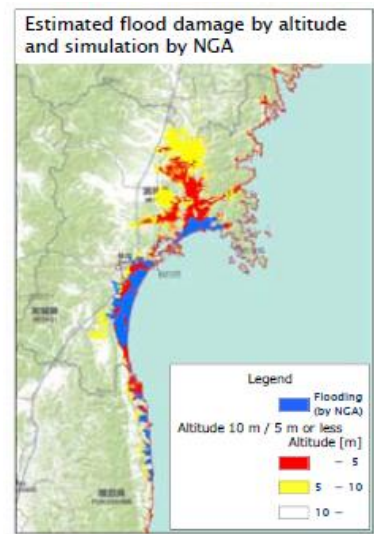

Most tsunami damages occurred in three prefectures in the Tohoku region

Estimated number of buildings damaged by the tsunami 1

About 270,000 buildings are needed to surveyed

\begin{tabular}{|c|c|c|c|}
\hline Pollecturser & $\sim \operatorname{sim}$ & silom & Total \\
\hline Iwate & 14.981 & 27.821 & 42,802 \\
\hline Miyagi & $\begin{array}{c}135.410 \\
(70.787)\end{array}$ & - & $\begin{array}{c}135,410 \\
(70.787)\end{array}$ \\
\hline $\begin{array}{l}\text { Fukushi } \\
\text { ma }\end{array}$ & 52.603 & 40,807 & 93.410 \\
\hline Total & $\begin{array}{r}202,994 \\
(138,371)\end{array}$ & 68,628 & $\begin{array}{r}271,622 \\
(206,999)\end{array}$ \\
\hline
\end{tabular}

Figure 3. Estimated number of buildings damaged by the tsunamis

\section{System design}

\subsection{Aim of the system}

The aim was to build a system to enable participation from anywhere in Japan. This requires a good network environment in order to secure a stable supply of human resources for the quick and efficient execution of the survey. Specifically, we designed a system to determine whether a building was washed away or not using the WEB-GIS by superimposing building data before the earthquake (building data) and aerial views after the earthquake (Figure 4).

4.3.2. Survey areas by roads. We intersected areas by roads, and each area was assigned a 13-digit ID number for each survey area (Figure 6). The 13-digit ID number was composed of a city, ward, or "cho" district code (5 digits), flood area code (4 digits), and survey area code for city, ward, and "cho" (4 digits). This made it easy to visually identify survey areas, and tally the results according to governmental jurisdictions.

\subsection{Fundamental concepts of design}

The following three points were established as the fundamental concepts of design. These concepts were determined based on discussions among information technology and psychology researchers. The discussions were centered on how to establish a mechanism that allowed investigators to intuitively conduct a survey without being stressed out in a cloud-based environment and how to motivate investigators in physically remote locations by providing visibility to other workers' progress.

4.3.1. Judgment patterns for washed-away buildings. Two categories were created for buildings that were washed away, either completely washed away or partially washed away. The purpose of this was to make it easy for an investigator to intuitively make a judgment (Figure 5). Despite our initial decision to include the unable to determine category during the development phase of this survey we decided against this. We were concerned that some investigators would categorize ambiguous cases to the unable to determine category if there was a discrepancy between the buildings being completely washed away or partially washed away. The purpose of this survey was to rapidly grasp the big picture of a wide-area disaster by spending only the minimum amount of time needed to assess building damage, instead of spending a large amount of time to precisely evaluate the degree of damage on each building. For this reason, the survey required investigators to always categorize all buildings into the categories of completely washed away or partially washed away.

4.3.2. Survey areas by roads. We intersected areas by roads, and each area was assigned a 13-digit ID number for each survey area (Figure 6). The 13-digit ID number was composed of a city, ward, or "cho" district code (5 digits), flood area code (4 digits), and survey area code for city, ward, and "cho" (4 digits). This made it easy to visually identify survey areas, and tally the results according to governmental jurisdictions. 


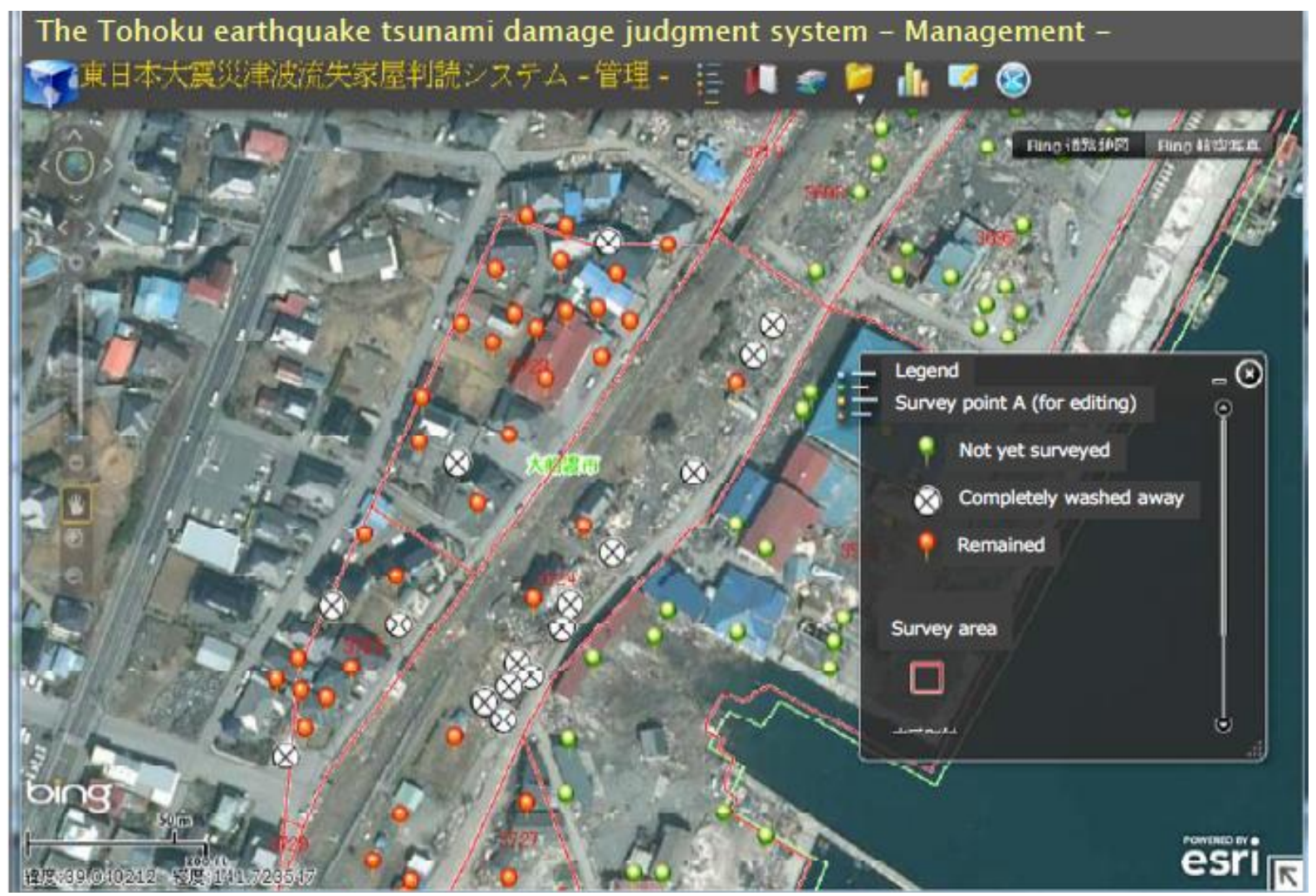

Figure 4. Screen design concept for the washed-away building judgment system using a Web-GIS

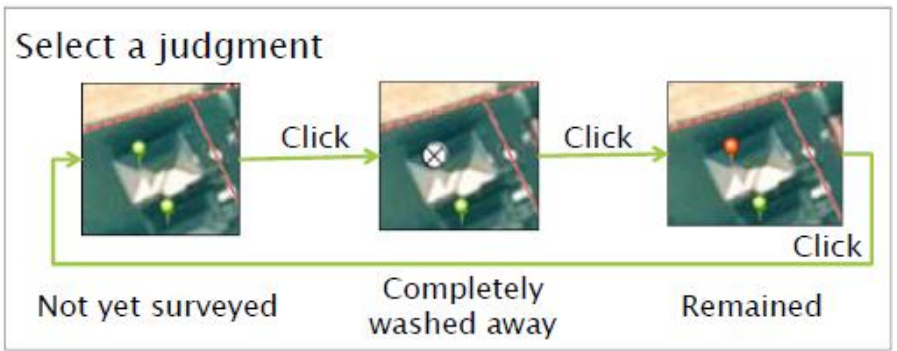

Figure 5. Judgment selection for building damage only by clicking

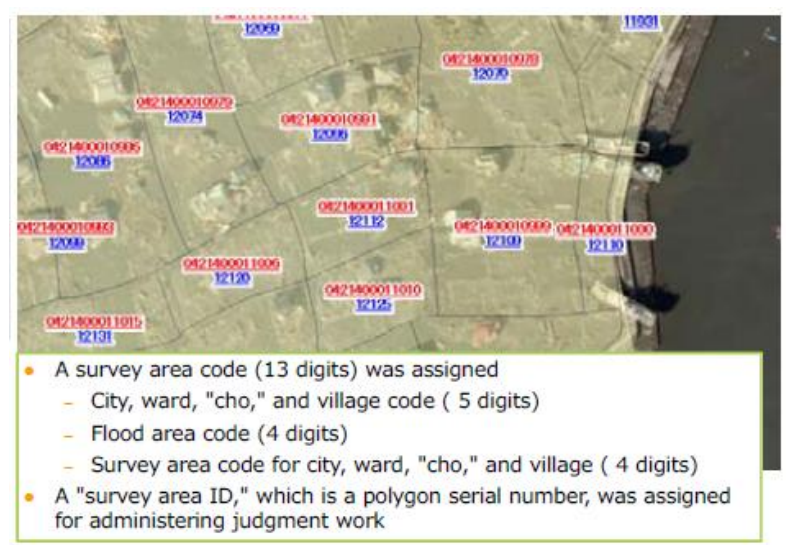

Figure 6. Code system for surveyed areas 
4.3.3. Progress of the survey to be viewed. Multiple investigators simultaneously conducted a survey using the WEB-GIS. We installed a feature to visualize the overall progress of the survey, which we hoped would motivate the investigators to conduct the survey (Figure 7).

\section{System configuration}
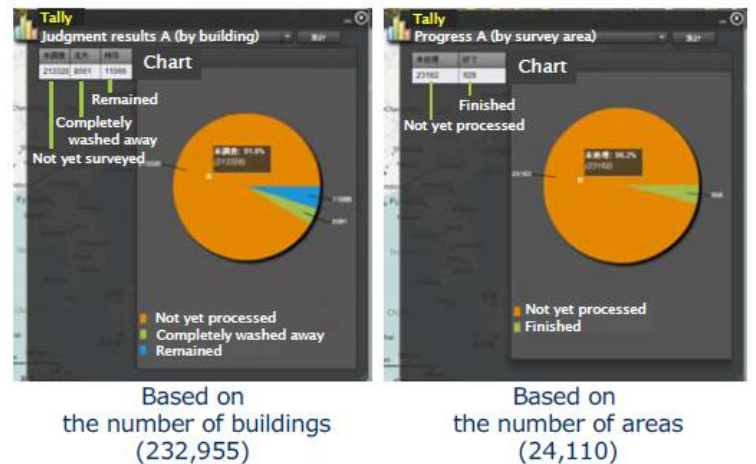

Figure 7. Tallying function to grasp progress of survey work

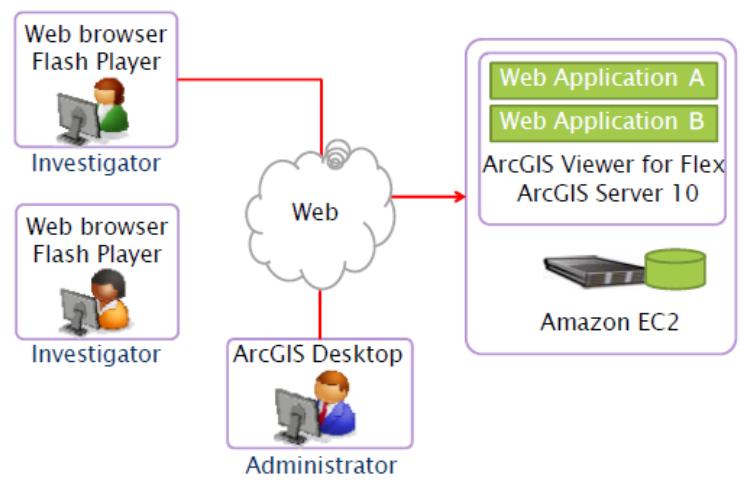

Figure 8. System Configuration

Configuration of the web application distribution environment involved five components (Figure 8).

1) Cloud Infrastructure. We selected a cloud infrastructure because it offered flexibility for changing the basic specifications, the ease of installation of the environment, and the potential for multiple accesses.

2) Middleware. We selected a middleware so that many people could simultaneously access, edit, and process the data over the spatial GIS system.

3) Application. We selected an application that allows the user to bidirectionally interact with the spatial GIS using a web browser.

4) Administrator of the web application. The administrator should be able to manage the progress of the survey and conduct a spatial analysis from their local environment, as well as exchange data with the cloud environment.

5) Investigator. Each investigator should have Flash Player, which is typically installed on most new personal computers. This serves as the operating environment for the personal computer, network environment, and web application.

\section{Project for system verification}

\subsection{Overview of the project}

To clarify issues that may arise while conducting the survey, in addition to considering an environment to efficiently and effectively tackle future disasters. To conduct a survey to identify buildings that were washed away in the three afflicted prefectures of Iwate, Miyagi, and Fukushima (excluding areas affected by a nuclear power plant accident because no aerial views were available for those areas). Moreover, to verify the effectiveness of this mechanism to identify buildings that were washed away using the WEB-GIS.

\subsection{Investigators}

Undergraduate students, graduate students, and researchers from five research groups in different universities were selected to conduct a study related to disaster prevention and disasters. Two independent investigators (different research groups) conducted a survey of the same area to verify an agreement between two survey results. This verification methodology is based on procedures used in psychological research studies. Each research group was sent an assignment table (in a Microsoft Excel format) that contained the names of the areas to be surveyed, the number of buildings in each area, and a blank column for names of the investigators. Each principal investigator (team leader: professor) was responsible for assigning the required jobs to co investigator and managing the progress of the survey.

\subsection{Instructions}

As instruction manual was prepared and is available online at all times. The 18-page instruction manual was prepared mainly by psychology researchers with assistance from information technology researchers and system developers. Two main principles were applied to prepare the instruction manual: 1. The number of pages should be kept minimal; and 2. The manual should be laid out according to the workflow (Figure 9).

\subsection{Instruction manual}

The instruction manual included the following chapters: Table of Contents

Purpose of This Project 
Chapter 1. Evaluation and Registration of Buildings Washed Away by the Tsunami

Step 1: Access the URL to login from the screen

Step 2: Display a layer for easy evaluation

Step 3: Evaluate buildings that were washed away

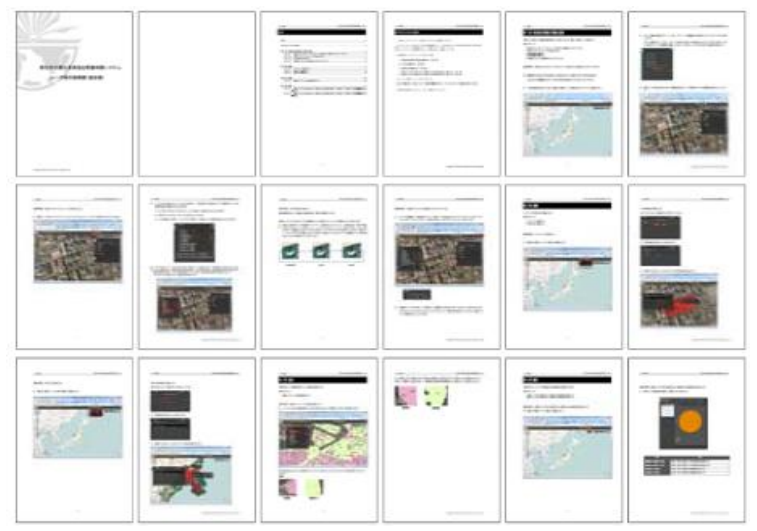

Figure 9. Instruction Manual (image)

Step 4: Complete evaluation or logout without completing the evaluation

Chapter 2. Search

Step 1: Search by area ID

Step 2: Search by city, cho, or village

Chapter 3. Progress

Step 1: Check the progress by survey area

Chapter 4. Tallying

Step 1: Tally the progress status for each survey area and building evaluation for each building

Step 2: Display the progress status for each survey area and building evaluation for each building

The four competencies that are required to carry out this project are listed in the "Purpose of This Project" and are based on the instructional design (ID). ID is a learning theory in psychology and educational technology, and it refers to the process that allows the investigators to clearly understand what they should learn while participating in this project. Users of the instruction manual said, "it is easy to understand both the big picture of the project and each job because my workflow is described in the order that I perform the tasks over a small number of pages." The following is the actual statement in the "Purpose of This Project:"

"Thank you very much for participating in this project. This is a disaster relief project involving multiple investigators to evaluate buildings that were washed away in disaster areas through shared and cooperative work. We ask that work be performed according to this manual. This manual is organized according to the following four competencies that are required to carry out this project: 1 . Evaluate and register buildings that were washed away by tsunami (Chapter 1), 2. Search by area (Chapter 2), 3. Check progress (Chapter 3), 4. Tally and display progress in each survey area and for each building evaluation (Chapter 4). In case you have a concern or doubt, please ask your team leader instead of making your own judgment. This is important in order to maintain the quality of the work."

\subsection{Instruction manual}

232,906 points were covered in 15,066 areas. There were 25,905 survey areas that were partitioned by roads. We subtracted 1,795 areas where surveys could not be conducted because airplanes could not fly due to the nuclear plant accident and 9,044 areas where there were no buildings (survey points); thus, resulting in 15,066 areas.

\section{Results of System verification}

\subsection{Results of concordant analysis}

Two investigators belonging to two different research groups conducted a survey of the same area. Of the 163,205 points that were evaluated, 90.9\% $(148,342$ points) were classified into the same category (either completely washed away or partially washed away) by the two investigators, and 9.1\% (14,863 points) were evaluated differently-the ratio of agreement was $90.9 \%$. A Pearson's chi-square test revealed that the ratio of agreement was statistically significant at $0.1 \% \quad(\mathrm{X} 2(1)=109305.7, \quad \mathrm{p}<.001)$. Another verification from a different viewpoint (similarity by symmetry) resulted in a $\Phi$ coefficient of 0.82 , which means that there was a strong positive correlation at a significance level of $0.1 \%(\Phi=0.82$, $\mathrm{p}<.001$ ) (Table 2).

\subsection{Lack of data and data accuracy}

An important issue to keep in mind is that there were no aerial views of the areas near the Fukushima Nuclear Power Plants since airplanes were not allowed to fly over these areas. Therefore, a survey using this method was not possible in these areas. Differences in the precision of the spatial information lead to discrepancies between the building information before the earthquake (building foundation data) and aerial views after the earthquake. In some instances these data discrepancies made it difficult to distinguish between a building that was in fact washed away and a building that appeared to be washed away. 
Table 2. Result of experimentation by two investigators (A and B)

\begin{tabular}{|c|c|c|c|c|}
\hline & & \multicolumn{2}{|c|}{ Result: Investigator " $\mathrm{B}$ " } & \multirow{2}{*}{ Total } \\
\hline & & Completely washed away & Remained & \\
\hline \multirow{2}{*}{$\begin{array}{c}\text { Result: } \\
\text { Investigator "A" }\end{array}$} & Completely washed away & $75,032(46.0 \%)$ & $6,027(3.7 \%)$ & $81,059(49.7 \%)$ \\
\hline & Remained & $8,836(5.4 \%)$ & $73,310(44.9 \%)$ & $82,146(50.3 \%)$ \\
\hline \multicolumn{2}{|r|}{ Total } & $83,868(51.4 \%)$ & $79,337(48.6 \%)$ & $163,205(100.0 \%)$ \\
\hline
\end{tabular}

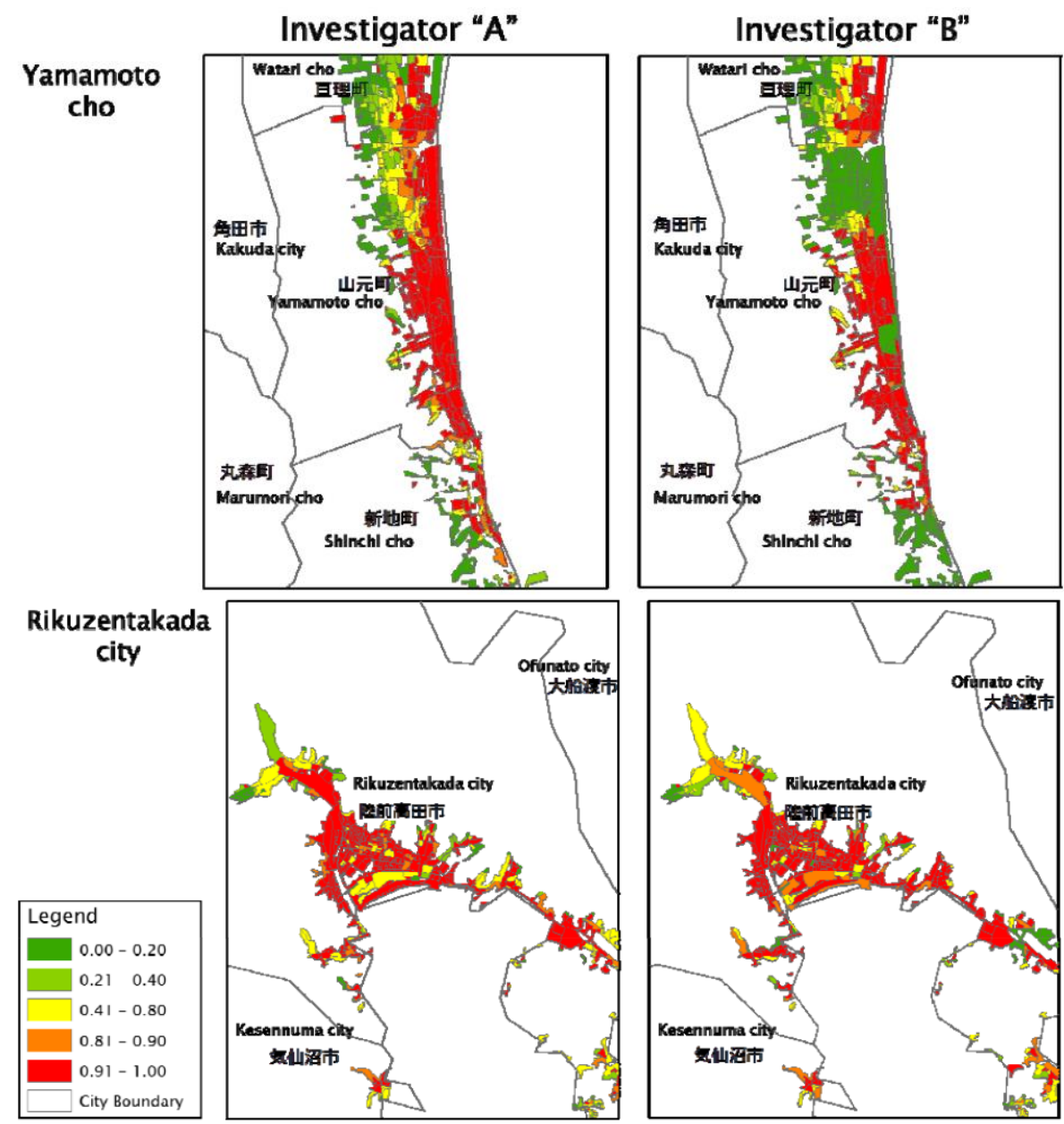

Figure 10. An Example of area where there was a discrepancy in judgment

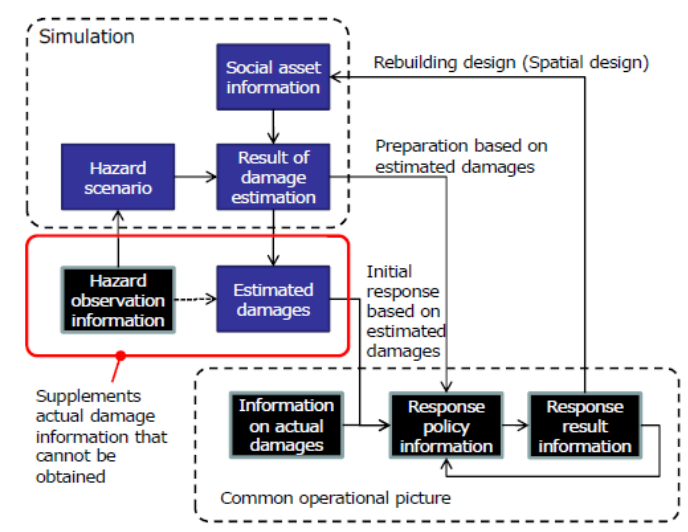

Figure 11. Information processing framework to achieve a comprehensive common operational picture 


\subsection{Operation management}

There were differences among the five research groups in the way the survey was conducted. Some groups followed the instructions, while others did not. Comparison of survey results conducted by different investigators revealed areas with a high degree of agreement and those with a low degree of agreement. This was likely due to personal bias in the criteria used by the investigators to make judgments according to the instructions (Figure 10).

\subsection{System interface}

We realized that it was necessary to develop an interface to minimize human errors in the operation management. In particular, there was an issue with investigator A mistakenly surveying an area that was assigned to investigator $\mathrm{B}$, which ended up overwriting the evaluation result of investigator $\mathrm{B}$. To rectify this issue, we added a link to the assignment management table that allows an area to be surveyed again and that takes the investigator automatically to the login screen for the appropriate survey area. Additionally, it became clear that a checking mechanism, using information such as a user ID, was required upon system login in the future.

\section{Information processing framework}

Based on our research team's experiences in this disaster, we proposed an information-processing framework as shown in Figure 11. Areas enclosed with dashed lines show a flow to obtain a common operational picture based on an assumption that actual damage situation is known. However, in the event that an actual damage situation cannot be determined, a flow to directly connect hazard observation information from hazard observation equipment was used. This was implemented in normal time, in response to the initial response system in order to minimize a delay in disaster response. It is important to utilize a simulation in normal times in order to connect hazard observation information to disaster response. In normal times, the relationship between a hazard scenario and social asset information (vulnerability) draws a possible damage result. With the aid of this framework, it is necessary to minimize a delay in disaster response by establishing an initial response system without an input of actual damage information and by having preparation based on possible damages. As disaster response progresses and actual damages gradually become known, approximated damages can be replaced with actual damages by area or by type of damage.

\section{Projected work}

Multiple people judged the effectiveness and viability of the cloud-based participatory WEB-GIS system to certify building damage due to the tsunamis. Although a web-based system can increase the participation of investigators, it is often difficult to guarantee the quality of investigation conducted. The effectiveness of this system was demonstrated with the assistance of psychological experiment methodology. This methodology is also expected to be effective in improving the reliability and appropriateness of data surveyed by multiple investigators, rather than being only an experimental model. In addition to our system functioning as an effective survey, it was a great achievement for us to generate a common operational picture among investigators and stakeholders through the sharing of the survey process on WEB-GIS. While this system was configured to be a judgment system, it is possible to adopt it to a wide variety of surveys depending on how categories are set for judgment and selection; therefore, it has promise as a highly versatile survey system. We aim to build a system with an even higher level of viability by solving the issues that we found in the areas of data, management, and systems.

\section{Acknowledgements}

This research was supported by JST (Japan Science and Technology Corporation), PRESTO (Precursory Research for Embryonic Science and Technology).

\section{References}

[1] S. Suzuki and H. Hayashi, "Spatial Exposure Analysis on Tokyo Metropolitan Earthquake Disaster", Journal of Disaster Research, Vol.5, No.1, Fuji Technology Press, Tokyo, Japan, February 2010, pp. 45-53.

[2] Y. Nawa, et.al., "Geography Network for Sharing Geospatial Information in Disaster Management", Journal of Disaster Research, Vol.5, No.1, Fuji Technology Press, Tokyo, Japan, February 2010, pp. 108-116.

[3] R. Kimura, M. Inoguchi, K. Tamura, Y. Nawa and H. Hayashi, "Implementation of a Web-Based and CloudBased Participatory GIS System to Certify Property Damage due to Tsunami”, International Conference on Information Society (i-Society) 2012 Proceedings, Institute of Electrical and Electronics Engineers (IEEE), UK and RI, June 2011, pp. 319-323. 